\title{
On the anomalous thermal conductivity of one-dimensional lattices
}

\author{
Stefano Lepri \\ Max-Planck-Institut für Physik Komplexer Systeme, Nöthnitzer Str. 38, D-01187 Dresden, Germany \\ lepri@mpipks-dresden.mpg.de \\ Roberto Livi $* \dagger$ \\ Dipartimento di Fisica dell'Università, L.go E.Fermi 2 I-50125 Firenze, Italy \\ livi@fi.infn.it \\ Antonio Politi i \\ Istituto Nazionale di Ottica, L.go E.Fermi 6 I-50125 Firenze, Italy \\ politi@ino.it
}

\begin{abstract}
The divergence of the thermal conductivity in the thermodynamic limit is thoroughly investigated. The divergence law is consistently determined with two different numerical approaches based on equilibrium and non-equilibrium simulations. A possible explanation in the framework of linear-response theory is also presented, which traces back the physical origin of this anomaly to the slow diffusion of the energy of long-wavelength Fourier modes. Finally, the results of dynamical simulations are compared with the predictions of mode-coupling theory.
\end{abstract}

Keywords: Heat conduction, Green-Kubo formula, Fourier modes

PACS numbers: 44.10.+i, 05.60.+w, 05.70.Ln

To appear in Europhysics Letters (1998)

An extremely idealized, but physically meaningful, model of an insulating solid is a set of $N$ atoms of mass $m$, arranged on a 1-d lattice with spacing $a$ and coupled by nonlinear forces. Denoting with $q_{l}$ the displacement of the $l$-th particle from its equilibrium position $l a$, the corresponding Hamiltonian reads

$$
H=\sum_{l=1}^{N}\left[{\frac{p_{l}}{2 m}}^{2}+V\left(q_{l+1}-q_{l}\right)\right],
$$

where $p_{l}=m \dot{q}_{l}$. If the chain is put in contact at its boundaries with two heat baths at different temperatures $T_{0}$ and $T_{0}+\Delta T$, a nonequilibrium stationary state arises, characterized by a non vanishing average heat flux $J$. The microscopic definition of $J$ is [1,2]

$$
J=\frac{1}{N} \sum_{l} j_{l}=\frac{a}{2} \frac{1}{N} \sum_{l} \dot{q}_{l}\left(f_{l+1}+f_{l}\right)
$$

where $f_{l}=-V^{\prime}\left(q_{l}-q_{l-1}\right)$ is the interaction force and $j_{l}$ represents the local flux at site $l$ (i.e. the sum of the fluxes of potential energy from its neighbours). The lattice thermal conductivity $\kappa$ can be defined through the Fourier law,

$$
\langle J\rangle=-\kappa \frac{d T}{d x},
$$

where $x=l a$ is the coordinate along the chain and $\langle\cdot\rangle$ denotes a time average in the stationary regime.

\footnotetext{
*also Istituto Nazionale di Fisica della Materia, Unità di Firenze

${ }^{\dagger}$ also Istituto Nazionale di Fisica Nucleare, Sezione di Firenze
} 
The task of statistical mechanics is to explain such a macroscopic law, starting from the microscopic interactions. Long time ago, Peierls proposed a theory based on the phonon scattering mechanism in the perturbative and close-to-equilibrium limits [3]. The common belief was thereby that a microscopic theory able to include the effects of disorder and/or nonlinearity should ensure the validity of Eq. (3) also in the case of strong anharmonicity and for systems far from equilibrium.

Nevertheless, the rigorous treatment of isotopically disordered harmonic chains showed that disorder alone is not sufficient for obtaining a reliable model: the thermal conductivity diverges as $\kappa \propto N^{1 / 2}$ [. Th. This result enforced the necessity to verify whether nonlinearities alone can eliminate such an anomaly. Recently, the problem has been reconsidered by resorting to nonequilibrium molecular-dynamics simulations. Among the many attempts, the most convincing results in favour of a finite $\kappa$ were obtained only for rather artificial systems, namely the ding-a-ling models [5] [6].

For more realistic systems, like the diatomic Toda chain a finite conductivity was found for small enough mass ratio [7], while the analysis of model (11) with the Fermi-Pasta-Ulam (FPU) potential

$$
V(y)=\frac{1}{2} m \omega_{0}^{2} y^{2}+\frac{1}{4} g y^{4},
$$

gives evidence of a power-law divergence $\kappa \propto N^{\beta}$ for $N \gg 1$ [8,2]. We have verified that this result is robust against changes in the number of thermostatted atoms, in the boundary conditions and in the type of heat baths (time-reversible as well as stochastic ones). More precisely, simulations reported in [2] with a large temperature gradient $\left(\Delta T=128\right.$ and $\left.T_{0}=24\right)$ reveal a crossover from $\beta \approx 0.45$ for $N<250$ to $\beta \approx 0.38$ for larger $N$, while new simulations made with $\Delta T=16$ and $T_{0}=80$ (Fig. 1) yield $\beta=0.37$ up to $N=2048$.

For sufficiently small $\Delta T$ and large $N$, the system is close to an equilibrium state at temperature $T=T_{0}+\frac{\Delta T}{2}$. One can then rely on the Green-Kubo formula [9]

$$
\kappa=\frac{1}{k_{B} T^{2}} \int_{0}^{\infty} C_{J}(t) d t
$$

where $C_{J}(t)=N\langle J(t) J(0)\rangle$ is the correlation function at equilibrium. We have numerically computed $C_{J}(t)$ for the FPU chain with Born-Von Karman boundary conditions, and initial conditions sampled according to the canonical Gibbs measure. In Fig. 2, we report the power spectrum $S(\omega)$ of the heat flux $J$ for $N=512$. In the low-frequency region there is a power-law divergence as $\omega^{-0.37}$, which corresponds to a decay of $C_{J}(t)$ as $t^{-0.63}$.

A crucial information for comparing these results with those obtained with heat baths comes from the spatiotemporal correlation function $C_{j}(l, t)=\left\langle j_{l}(t) j_{0}(0)\right\rangle$ of the local flux. Indeed, the peaks shown in Fig. 3 indicate that energy propagates at constant velocity $v_{p}$. Therefore, one can estimate the dependence of $\kappa$ on $N$ from the asymptotic behaviour of $C_{J}(t)$, by restricting the integral in (5) to times smaller than the "transit time" $N / v_{p}$. This amounts to ignoring all the contributions from sites at a distance larger than $N$. With the above estimate of $C_{J}$, one obtains that $\kappa \propto N^{0.37}$, in agreement with the direct estimate obtained from the non-equilibrium simulations in Fig. 1.

Despite the remarkable consistency of the numerical results, the scenario is quite unsatisfactory on a theoretical ground, especially because there is neither a clue about the very reason of the observed anomalous transport, nor an explicit estimate of the measured exponent. In the following, we present a possible explanation based on a kind of hydrodynamic approach with long-wavelength modes interacting with a stochastic-like reservoir.

In fact, it has been observed [10] that even at high energies, where the FPU-system is definitely nonintegrable, the energy of its long-wavelength Fourier modes diffuses very slowly (with respect to their period). Accordingly, they may effectively act as undamped transport channels, a feature that reflects itself in the relaxation of fluctuations as measured by $C_{J}(t)$. Our goal is now to obtain an estimate of the asymptotic behaviour of $C_{J}(t)$, by describing the relaxation close to equilibrium of the Fourier-mode amplitudes $A_{k}$ and $B_{k}$, defined by

$$
q_{l}=\frac{1}{\sqrt{N}} \sum_{k=1}^{N / 2}\left[A_{k} \cos \left(\frac{2 \pi k a}{N} l\right)+B_{k} \sin \left(\frac{2 \pi k a}{N} l\right)\right],
$$

where we have made the assumption that the zero-mode $A_{0}$ is identically zero. The customary approach would be to write stochastic equations for $A_{k}$ and $B_{k}$. The general strategy involves projection on the subspace of such (slow) variables and yields linear Langevin equations with memory terms [9]. If we assume that a separation of proper time scales is possible, the former reduce to Markovian equations [1],

$$
\ddot{A}_{k}+\gamma_{k} \dot{A}_{k}+\tilde{\omega}_{k}^{2} A_{k}=\xi_{k} \quad ; \quad \ddot{B}_{k}+\gamma_{k} \dot{B}_{k}+\tilde{\omega}_{k}^{2} B_{k}=\eta_{k}
$$


where $\gamma_{k}$ are phenomenological relaxation rates, $\xi_{k}$ and $\eta_{k}$ are mutually independent, Gaussian, random and white processes 12 . Nonlinear effects are taken into account by renormalization of the bare dispersion relation as 10

$$
\tilde{\omega}_{k}=\alpha \omega_{k}=\alpha \cdot 2 \omega_{0} \sin \left(\frac{\pi k a}{N}\right),
$$

i.e. by renormalizing the sound velocity from $v=a \omega_{0}$ to $\tilde{v}=\alpha v$, where, for the parameters of Fig. 1, $\alpha=\sqrt{\left[1+2(1+0.72 \varepsilon)^{1 / 2}\right] / 3}$ and $\varepsilon(T)$ is the internal energy [10].

A first confirmation that transport is basically determined by the low $k$-modes comes from the observation that $\tilde{v}$ is remarkably close to $v_{p}$ : for the parameter values considered in Fig. $3, \tilde{v}=2.38$, while $v_{p}=2.47$.

Let us now split $V$ into its harmonic and anharmonic parts and consequently write the flux (2) as $J=J_{H}+J_{A}$. From Eq. (6) one obtains

$$
J_{H}=\frac{m}{N} \sum_{k=1}^{N / 2} c_{k} \omega_{k}\left(A_{k} \dot{B}_{k}-\dot{A}_{k} B_{k}\right)
$$

where $c_{k}=d \omega_{k} / d k$ is the bare phase velocity. For a strongly anharmonic system, like the one we consider here, we do not expect $J_{A}$ to be negligible. Nevertheless, we shall now argue that the leading asymptotic behaviour of $C_{J}$ can be estimated by computing the autocorrelation of $J_{H}$ alone. We limit ourselves to the FPU case (雨), for which $J_{A}$ is a sum of fourth order terms coupling modes with indices $k, k^{\prime}, k^{\prime \prime}, k^{\prime \prime \prime}$. By virtue of Eq. (7), each low- $k$ component oscillates approximatively at a frequency that is an algebraic sum of $\tilde{\omega}_{k}, \tilde{\omega}_{k^{\prime}}, \tilde{\omega}_{k^{\prime \prime}}$ and $\tilde{\omega}_{k^{\prime \prime \prime}}$. It is then reasonable to assume that rapidly oscillating terms will be relevant only for short times, while the asymptotic dominant contributions originate from the resonant terms. This fact, together with selection rules, require that only terms fulfilling the constraints

$$
\begin{gathered}
k+k^{\prime}+k^{\prime \prime}+k^{\prime \prime \prime}=0 \\
\tilde{\omega}_{k}+\tilde{\omega}_{k^{\prime}}-\tilde{\omega}_{k^{\prime \prime}}-\tilde{\omega}_{k^{\prime \prime \prime}}=0
\end{gathered}
$$

must be retained. For small $k$, we can linearize the dispersion relation in the second of Eqs. (10) obtaining

$$
J_{A} \approx J_{H} \frac{g}{m \omega_{0}^{4}} \frac{1}{N} \sum_{k} \omega_{k}^{2}\left(A_{k}^{2}+B_{k}^{2}\right)
$$

In order to evaluate the contribution of $J_{A}$ to $C_{J}$, let us first notice that the former is the product of two time-dependent terms: $J_{H}$ and the sum $\mathcal{S}=\sum \omega_{k}^{2}\left(A_{k}^{2}+B_{k}^{2}\right)$. Any given $k$-contribution in the expression of $J_{H}$ (see Eq. (9)), is correlated with only one (with the same $k$ ) out of the $N$ addenda of the sum in Eq. (11). Accordingly, in the thermodynamic limit, we can consider $\mathcal{S}$ as uncorrelated with $J_{H}$. Moreover, since $\mathcal{S}$ is the sum of uncorrelated positive terms, we can substitute it with its average value and write

$$
J \approx J_{H}\left(1+\frac{4 g}{m^{2} \omega_{0}^{4}} \mathcal{U}(T)\right)
$$

where we have also taken into account energy equipartition in its generalized form, i.e. $m \omega_{k}^{2}\left\langle A_{k}^{2}\right\rangle=m \omega_{k}^{2}\left\langle B_{k}^{2}\right\rangle=$ $\mathcal{U}(T)$, for some function $\mathcal{U}$ independent of $k$. We numerically checked the reliability of our approximations by first comparing expression (11) with $J_{A}$ and then by verifying that the autocorrelation functions of $J_{H}$ and $J$ are proportional.

Let us compute the autocorrelation of $J_{H}$. Using Eqs. (7), one finds that the new variables $W_{k}=A_{k} \dot{B}_{k}-\dot{A}_{k} B_{k}$ satisfy the Langevin equation

$$
\dot{W}_{k}=-\gamma_{k} W_{k}+\zeta_{k} .
$$

In the limit of small $\gamma_{k}, \zeta_{k}$ is a Gaussian and delta-correlated random process, so that for large $N$

$$
\left\langle J_{H}(t) J_{H}(0)\right\rangle=\frac{4}{N^{2}} k_{B} T \mathcal{U}(T) \sum_{k} c_{k}{ }^{2} e^{-\gamma_{k} t} \approx \frac{1}{N} \frac{2 a}{\pi} k_{B} T \mathcal{U}(T) \int_{0}^{\pi / a} d k c^{2}(k) e^{-\gamma(k) t}
$$


Due to the conservation of total momentum, one expects $\gamma_{k}$ to vanish for vanishing $k$. By drawing an analogy with the known nonanalytic behaviour of dispersion relations of low-dimensional fluids [13], we may assume $\gamma(k) \approx \nu k^{\mu}$, to leading order in $k$ [14. We also suppose that $\mu>1$ consistently with the observed fact that dissipation occurs on time scales much longer than the proper period. Since the conduction properties are determined by low- $k$ modes, we can approximate Eq.(14) by expanding $c(k)$ around $k=0$ so that

$$
\left\langle J_{H}(t) J_{H}(0)\right\rangle \propto \frac{1}{N} \frac{v^{2} k_{B} T \mathcal{U}(T) a}{(\nu t)^{\frac{1}{\mu}}}\left[1+\mathcal{O}\left(t^{-\frac{2}{\mu}}\right)\right] .
$$

The power-law decay of $C_{J}(t)$ implies that $\kappa$ should diverge as $N^{1-1 / \mu}$.

The key point is then the evaluation of $\mu$. In the case of fluids, this is generally accomplished by resorting to a self-consistent mode-coupling approximation 14]. In the present context, it is reasonable to conjecture, on the basis of Eqs. (7), that the behaviour of low- $k$ modes can be assimilated to that of hydrodynamic modes in a 1d fluid, at least at such high temperatures (see [11] for a more detailed analytical discussion). One should then expect $\mu=5 / 3$ [15], thus yielding $C_{J}(t) \propto t^{-3 / 5}$ and $\kappa \propto N^{2 / 5}$. This prediction is compared with the numerical results in the inset of Fig. 2. The discrepancy with the best-fit value (0.37) can be definitely attributed to finite-size effects. As long as the above conjecture proves correct, our numerical results provide the most striking confirmation of the mode coupling predictions.

The generality of our arguments suggests that any nonlinear 1-d model with an acoustic spectral component should exhibit the same anomaly. It has however to be admitted that the mentioned Toda model in which an optical branch is also present [7] is qualitatively different.

Finally, we observe that there is no reason for expecting the same phenomenon not to occur also in higher dimensions, independently in each acoustic branch. On the other hand, the exponent $\mu$ may well depend on $d$ and, moreover, the integral in Eq. (14) has to be weighted with the phononic density of states, which, for small $k$, vanishes as $k^{d-1}$. One can conjecture that such a mechanism does not introduce any divergence in the conductivity for $d=3$, while a logarithmic divergence should be observed for $d=2$. This prediction provides a unified explanation for both the anomalous transport of 1-d systems and the finite conductivity of 3-d lattices.

[1] Ph. Choquard, Helvetica Physica Acta, 36, 415 (1963).

[2] S. Lepri, R. Livi, A. Politi, Physica D in print.

[3] R.E. Peierls, Quantum theory of solids, Oxford University Press, London (1955).

[4] J.B. Keller, G.C. Papanicolaou, J. Weilenmann, Comm. Pure and App. Math. Vol. XXXII, 583 (1978).

[5] G. Casati, J. Ford, F. Vivaldi, W.M. Visscher, Phys. Rev. Lett. 52, 1861 (1984).

[6] T.Prosen, M. Robnick, J. Phys. A 253449 (1992).

[7] E. A. Jackson, A. D. Mistriotis, J. Phys. Condens. Matter 11223 (1989).

[8] S. Lepri, R. Livi, A. Politi, Phys. Rev. Lett. 78, 1896 (1997).

[9] R.Kubo, M.Toda, N. Hashitsume, Statistical Physics II, Springer Series in Solid State Sciences, Vol. 31 (1991).

[10] C. Alabiso, M.Casartelli, P.Marenzoni, J. Stat. Phys. 79, 451 (1995).

[11] S. Lepri, "Relaxation of many-body hamiltonians in one dimension", preprint (1998).

[12] The statistical independence of the modes stems from translational invariance, as it can be shown, by computing, e.g., $\left\langle A_{k}(t) B_{k^{\prime}}(0)\right\rangle$ from Eq. (6).

[13] We are indebted to an anonymous referee for having pointed out this connection.

[14] Y. Pomeau, R. Résibois, Phys. Rep. 1963 (1975).

[15] M.H. Ernst, Physica D 47198 (1991) 


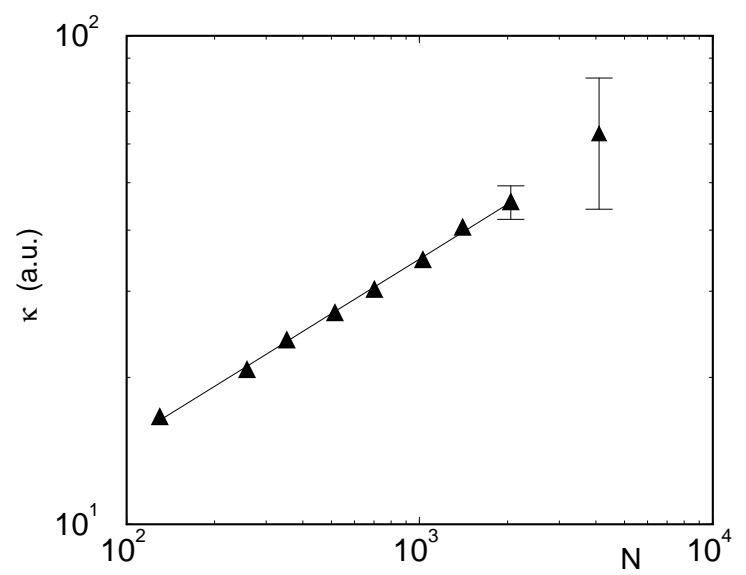

FIG. 1. Divergence of the thermal conductivity with the system size for the FPU chain $\left(m=\omega_{0}=1\right.$, $g=0.1$ ) as obtained from nonequilibrium molecular dynamics (see Refs. [8, 2] for details). Statistical errors are displayed only when larger than the symbols' size.

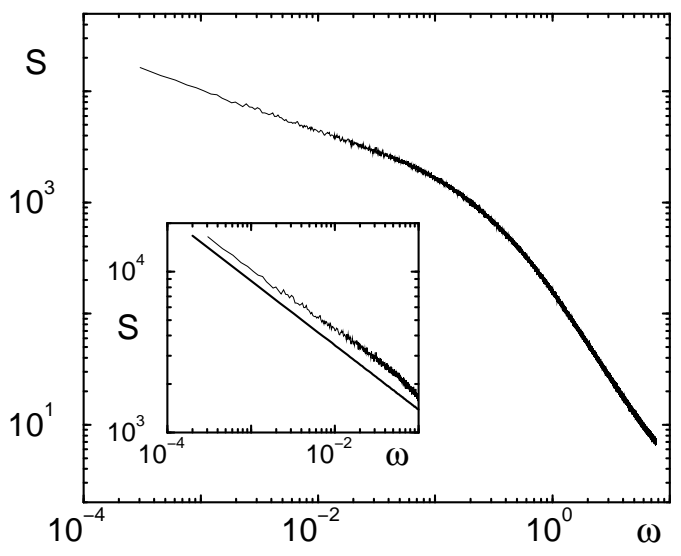

FIG. 2. Power spectrum $S(\omega)$ (in arbitrary units) of the global flux for the FPU chain (same parameters as in Fig. 1) with $N=1024$ at temperature $T=110.7$. The curve results from an average over 1400 independent initial conditions. A blow-up of the low-frequency region is reported in the inset: the straight line corresponds to the $1 / \omega^{2 / 5}$ behaviour. 


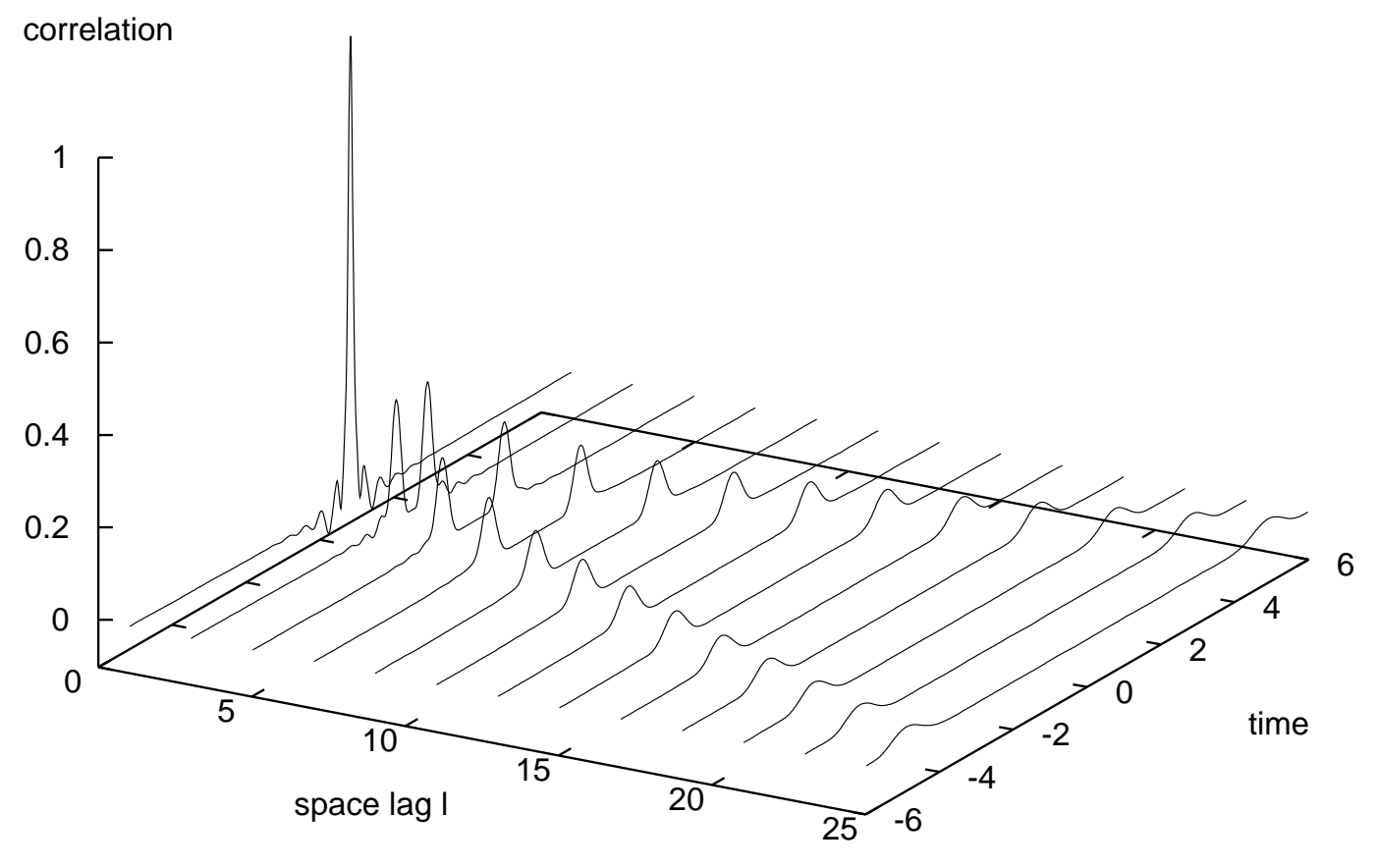

FIG. 3. The (normalized) correlation $C_{j}(l, t)$ of the local flux for the FPU model with $N=1024$ (same parameters as Fig. 1). 